\title{
Zur Casuistik und Aetiologie der Wanderleber.
}

Von

\author{
Dr. F. N. Winklex, \\ prakt. Arzt in Dresden.
}

Bei der Seltenheit der Wanderleber halte ich es für geboten, zu den drei bisher bekannten Fällen einen neuen hinzuzufügen, den ich mehrere Jahre hindurch in Jena in Beobachtung hatte und der sich vor den bisherigen durch ein neues Symptom auszeichnet.

Christiane Juch, $29 \mathrm{Jahr}$, in Jena Wärterin und vorher Magd, war bisher stets gesund. - Erstes Kind: 1862. Schon damals Hängebauch; auch nach der Geburt blieb der Leib schlaff und propendirend. Z $\mathrm{Z}_{\text {weites }} \mathrm{Kind}$ : Ende Sommer 1868. Sehr starker Hängebauch; ausserdem während der ganzen zweiten Hälfte der Schwangerschaft ein sehr quälender Hustenreiz mit häufigen Hustenanfällen, während welcher in beiden Hypochondrien, zumal rechts, schon die nämlichen Schmerzen eintraten, wie später. Dasselbe war der Fall bei tiefem Luftholen.

Etwa 4 Wochen nach der Niederkunft bekam Pat. beim Heben einer schweren Last stechende Schmerzen im rechten Hypochondrium, die von links nach rechts zogen und hier sich schliesslich fixirten. Dabei öfters Aufstossen, Uebelkeiten, kalter Schweiss, verfallenes Gesicht. Im Stehen bestand mehr Schmerz als im Liegen, besonders aber in linker Seitenlage. Dieser Zustand dauerte 3 Tage an; aber auch nachher blieb Pat. nicht schmerzfrei, obwohl dies je nach Tagen wechselte. Besonders traten diese Schmerzen im rechten Нypochondrium auf bei schwerem Heben, sowie bei jeder Erschütterung, wie Treppenlaufen, Springen, Gestossenwerden, Straucheln; ebenso 
auch beim Rückwärtsbiegen. Erleichterung trat stets ein durch Vornüberbiegen, Stiitzen des Leibes mit den Händen. Patientin hatte schon früher des Hängebauchs wegen eine Bauchbinde erhalten, dieselbe jedoch nur locker angelegt und deshalb keine Erleichterung gehabt.

Mitte November 1868 - die später folgenden Lebermaasse beziehen sich auf diesen Termin - legte ich eine Binde recht fest an, so dass besonders der Unterbauch fest comprimirt wurde. Darauf blieb Patientin völlig schmerzfrei, bis sie October 1869 die Binde ablegte, weil sie nur leichte Arbeit hatte. Aber auch jetzt befand sie sich wohl bis März 1870, wo sich nach einer schweren Arbeit beim Waschen die alten Schmerzen, und fast noch intensiver als früher einstellten; allmälig entwickelte sich auch ein mässiger Icterus, der etwa 4 Wochen anhielt. Patientin hatte dic letzte Zeit im Bette zugebracht und dann von Nenem die bewährte Binde an- und seitdem auch nicht wieder abgelegt. Bis Ostern 1872 hat sie sich seitdem völlig wohl gefühlt

Der Befund, wie ich ihn November 1868 aufgenommen, war auch später noch, sobald die Binde eine Zeit lang abgelegt worden, im Wesentlichen stets der gleiche und lautete wie folgt:

Durch die äusserst dünnen und schlaffen Bauchdecken konnte man widerstandslos fast in sämmtliche Tiefen der Bauchhöhle eindringen und fand die rechte Hälfte der Bauchhöhle eingenommen von einem umfangreichen, derben, verschiebbaren Tumor, der durch seinen unteren Rand sofort an die Contouren der Leber erinnerte und zwar war der Befund sofort ein so prägnanter, dass die Sachlage mir durchaus klar erschien, obwohl mir damals die zwei italienischen Fälle von Wanderleber noch unbekannt, und der Meissnersche noch nicht veröffentlicht waren. Versuchte man die Leber so zu umfassen, dass 4 Finger an die untere, der Daumen an die obere Fläche zu liegen kamen, so drang man ohne Weiteres bis an die Schwimmhaut in die Tiefe. Darauf gelang es, einen grossen Theil der unteren Leberfläche zu betasten, so dass man. Furchen deutlich erkennen konnte, weniger die Gallenblase, die ebensogut ein Darmstück sein konnte, denn man fühlte an dieser Stelle eine Art von Gleiten dünner sehlüpfriger Membranen in der Weise, wie sich leere Darmschlingen neben oder hinter dem Uterus bei combinirter Untersuchung anzufühlen pflegen. Jedenfalls war aber die Gallenblase nur wenig gefiillt. Zwischen Rippesbogen und Leber konnte man gleichfalls hoch in die Excavatio diaphragmatica hinanfdringen und hier unter geringer Hinabdrängung der Leber das offenbar sehr verlängerte Lig. suspensorium hepatis als eine scharf gespannte diunne Platte erkennen. Die Leber selbst hatte durchaus normale Consistenz, normale Gestalt und normalen scharfen vorderen Rand. Die Beweglichkeit des Organs war sehr bedeutend nach links und nach oben: in letzterer Richtung konnte die Leber vollständig reponirt werden, so dass ihr vorderer Rand vollständig mit dem Rippenbogen abschloss; dabei war, was hier gleich erwähnt sei, ihre obere Grenze (wahre 
Lebergrenze) um etwa $2 \mathrm{Cm}$. gestiegen. Suchte man die Leber nach abwärts oder nach links zu drängen, so liessen sich jene oben beschriebenen Schmerzen öfters hervorrufen.

Nachdem ich hierauf die Patientin hatte aufstehen und mehrere Male auf und abgehen lassen, nahm ich in horizontaler Rüickenlage folgende Maasse auf:

I. Wahre Lebergrenze: (Kuppel des Diaphragma).

L. parast. - unterer Rand der 5. Costa.

L. papill. - oberer Rand der 6. "

L. axill. schneidet die $8 .$,

II. Untere Lungengrenze: ca. $4 \mathrm{Cm}$. tiefer

in Lin. papill. schneidet 7. Costa.

in Lin. axill. " $8 .$,

III. Untere Lebergrenze ist unter dem Rippenbogen

in L, parast. $8 \mathrm{Cm}$.

in L. pap. 6 "

in L. axill. 1 ,

IV. Ganze Leberhöhe:

in L. parast. $13 \mathrm{Cm}$.
in L. pap. $16 \quad "$
in L. axill. $10 "$,

Ueberragt nach links die Lin. med, um 6,5 Çm.

Im Stehen fällt die obere (wahre) Grenze um $3 \mathrm{Cm}$; um etwa ebensoviel sinkt die untere. Dabei reicht die Leber bis anf die Crista, nach vorn bis auf die Spin. ant. sup. Bis zu dieser ist von hinten her überall leerer Schall, der aber in linker Seitenlage sofort durch Darmschall in weitem Umfange hinten bis zum Quadratus lumborum ersetzt wird.

Hinten reicht im Stehen der volle Lungenschall $4 \mathrm{Cm}$. nach rechts von der Wirbelsärle bis ins Niveau des 12. Proe. spinos. und erst hier beginnt die wahre Lebergrenze (bei tiefer Percussion). Nach fester Anlegung der Bauchbinde reichte im Stehen der vordere Leberrand kaum einige Centimeter unter den Rippenbogen.

Die Leber war somit 1) in toto herabgesunken; 2) um eine Queraxe nach vorn gehebelt; 3) beiderlei Bewegungen waren am ausgiebigsten am linken Lappen, am geringsten am rechten Leberrande zu Stande gekommen, so dass noch eine dritte Bewegung gleichzeitig erfolgt war, nämlich Drehung um eine im rechten Leberrande belegene Antero-Posterioraxe. Diese dreifache Richtung der Bewegung konnte man recht deutlich während der manuellen Repositions- und darauf folgender Dislocationsversuche erkennen; sie geht auch hervor aus den Zahlen sub. III und IV. Die Zahlen sub. I und II zeigen, dass die Leber sammt 
Diaphragma herabgesunken war, letzteres jedoch nicht in demselben Verhältniss wie die Leber, so dass ein Theil der Excursion der Leber auf die, wenigstens für das Lig. suspensorium durch Palpation gefundene Verlängerung der Leberligamente geschoben werden muss.

Bei einem hochgradigen Herabgleiten der Leber an der vorderen Bauchwand muss es schliesslich zu einer Knickung des Ductus choledochus, sobald aber noch andere Richtungen für die Leberdislocation in Frage gelangen, nebenbei noch zu einer Art von Torsion dieses Gallenganges mit mehr oder minder bedeutender Verengerung seines Lumens kommen, der Effect ist dann Gallenstauung, und unter Umständen Stauungsicterus. Diese Erklärung nehme ich in dem Falle $J$ uch für den Icterus.im Frühjahr 1870 mit grosser Wahrscheinlichkeit in Anspruch, weil dieser Icterus gerade mit einem erneuerten Anfalle von Leberdislocation direct zusammenhing. Die schmerzhaften Anfälle zu erklären ist eben so schwer, wie überhaupt die schmerzhaften Sensationen innerhalb der Peritonäalhöhle. Die Zerrung am Lig. suspensorium oder coronarium halte ich nicht für den Hauptgrund, denn es giebt genug Analogien, in welchen bei Zerrung nicht complicitirter peritonäaler Falten keinerlei Schmerzen eintreten, ebensowenig die etwaige Verlegung des Duct. choledochus, denn auch der gewöhnliche Icterus catarrhalis verläuft ohne Schmerzen; ich würde die Quelle der Schmerzen am ehesten in die zum Hilus hepatis laufenden Stränge verlegen, die bei Nachgiebigkeit der anderen Ligamente Zerrung sowohl, als auch Torsion erleiden und als nervenreiche Bahnen am meisten noch darauf in Form von Schmerz reagiren werden. Die Schmerzanfälle selbst ähnelten mitunter aufs Haar der Gallenstein-Kolik und nur die so sehr imponirende Dislocation der Leber, sowie das plötzliche Aufhören aller Erscheinungen nach erfolgter Reposition des Organs bedingten es, dass keine Verwechselung aufkommen konnte.

Was die Aetiologie der Wanderleber anbetrifft, so muss auf die bisher bekannten drei Fälle recurrirt werden. Meissner beschrieb ${ }^{\text {) }} 1869$ einen dritten Fall und stellte ihn mit den schon bekannten 2 Fällen von Cantani, 1866, und Piatelli, 1868, zusammen.

Die bekannten 4 Fälle betreffen Frauen, bei denen nach

1) Schmidt's Jahrb. Bd. 141. 
Schwangerschaften mehr oder minder starker Hängebauch zurückblieb.

Cantani sah sich veranlasst, für die Aetiologie einen Einfluss der Gravidität oder Geburt in Anspruch zu nehmen, etwa derart, dass auf Grund einer durch den hochschwangeren Uterus bedingten Rückwärtsdrängung der Leber die Leberligamente entzündet, gezerrt, gedehnt und dann nach der Geburt immer länger ausgezogen würden. Dagegen opponirt Meissner: 1) trotz der Häufigkeit der Schwangerschaft sei Wanderleber überaus selten, 2) Perihepatitis erzeuge keine Laxheit der Befestigung, sondern im Gegentheil Zunahme der Fixation, 3) der Uterus könne die Leber nicht nach hinten, wohl aber nach vorn drängen, 4) solcher Druck müsste während seines Bestehens unbedingt ernste Erscheinungen hervorgerufen haben, was in der Wirklichkeit nicht der Fall war.

Me issner nimmt als aetiologisches Moment für die abnorme Beweglichkeit der Leber eine Verlängerung der Ligamente (ein „Mesohepar") als eine ursprüngliche Bildungsanomalie an.

Die Meissner'schen Gegengründe ad 1) und 2) sind durchaus richtig. Dagegen 3) kann meinerseits nicht concedirt werden und zwar aus Gründen die ich sofort näher entwickeln werde; somit kommt auch ad 4) in Wegfall.

Es wird sich empfehlen, die Verhältnisse innerhalb der Bauchhöhle etwas genauer ins Auge zu fassen.

Im normalen Zustande reicht die Kuppel des Diaphragma bis ins Niveau der 5. Rippe oder etwa des 9. Rückenwirbels. Ihr Gipfel liegt mehr vorn, und zwar weil die Richtung des intraabdominellen Druckes, abgleitend von der Convexität der Wirbelsäule, nach vorn mehr und oben gelenkt wird. Die Kraft, welche bei etwaigen Dislocationen der Leber spielen dürfte, lässst sich mechanisch ausdrücken als Lebergewicht minus intraabdomineller Druck; sind beide gleich, so ist das Resultat Null und die Leber bleibt ruhig liegen. In dem Maasse aber, als der Druck abnimmt, nimmt der Effect des Lebergewichtes zu. Ersteres, d. h. Ruhestellung der Leber scheint unter normalen Verhältnissen zu bestehen, so dass die mechanische Aufgabe der Ligamente eine höchst geringe ist und wohl nur seitliche Excursionen verhindert, da ja bei den meisten Körperbewegungen der intraabdominelle Druck nicht unbeträchtlich vermehrt, die Leber somit nur um so fester in die Kuppel des Zwerchfelles hineingedrückt und in ihren seit- 
lichen Excursionen schon durch die Trichtergestalt des Zwerchfells verhindert wird.

Für gewöhnlich wird somit auch die Länge der Leberligamente völlig irrelevant sein, und selbst bei bedeutender Länge derselben, gleichviel ob erstere eine primäre Bildungsanomalie oder durch irgendwelche Zustände erst secundär entstanden sei, wird die Leber keine wesentlichen Dislocationen eingehen können.

Anders wird die Sachlage aber sofort, sobald durch bedeutende Abnahme des intraabdominellen Druckes die wesentlichsten Fixationsmittel für die Leber verloren gehen. Die Leber oder Milz bekommen unter diesen Umständen die Tendenz zum Descensus, der um so leichter zu Stande kommen muss, je schwerer das Organ. Leberdescensus bei Hängebauch ist demnach ein völliges Analogon des Descensus uteri bei Erschlaffung des Beckenbodens. Der nächste Effect ist bei dieser Sachlage das tiefe Herabtreten der Leber sammt Zwerchfell, und die etwaigen Beschwerden werden keine anderen sein, als sie überhaupt bei Hängebauch vorkommen, so Gefühl von Druck, Schwere, Vollsein, Zerren im Unterleibe. Auf dieser Stufe scheint mir der Meiss ner'sche Fall gewesen zu sein. - Hält nun dieses Stadium längere Zeit an, so ist es ganz offenbar, gleichviel wie lang die Ligamente vorher waren, dass ein Organ von 2 Kilo im Minimum an Gewicht allmälig eine Dehnung dieser Bänder herbeifübren muss: in erster Linie wird das L. suspensor. verlängert, der linke Lappen senkt sich am meisten (d. i. obige Axendrehung um eine Anteroposterior-Axe), und weil in einem so schlaffen Bauche im Stehen eben Alles nach vorn gleichzeitig gravitirt, so wird auch das Lig. coronarium verlängert, so dass die Leber längs der vorderen Bauchwand herabgleitet. Viel weniger nachgiebig, als die eben erwähntèn Bänder, sind alle zum Hilus hepatis ziehenden Stränge, und von hier aus müssen bedeutende Zerrungs-, und wie schon oben erwähnt, selbst bis zum Stauungsicterus führende Knickungsund Torsionssymptome sich entwickeln. Diesen höchsten Grad von Entwickelung secundärer Erscheinungen zeigte mein Fall.

Somit muss für Entstehung der Wanderleber als besonders bedeutungsvolles Moment ein schlaffer Hängeba uch angesehen werden, wie ihn Schwangerschaften mitunter erzeugen. Dass nicht in jedem solchen Falle der stets nothwendig eintretende Leberdescensus zu Torsionserscheinungen der Hilusstränge führt, lässt sich ganz gut aus individuellen Schwierigkeiten für Eintritt 
des höchsten Grades des Descensus erkiären, insofern als die Ligamente bei manchen Individuen nicht nachgiebig und sehr wenig dehnbar sein mögen, somit die Leber nicht ganz tief herabgleiten lassen. Oder der Hängebauch ist, anstatt schlaff, durch besondere Füllung oder Entwickelung der Därme oder durch besonders starke Fettansammlung am Omentum ein straffer geworden, so dass die Leber wieder eine erkleckliche Stütze findet.

Aus diesen Erwägungen geht für die Therapie die einzige. Indication hervor, den intraabdominellen Druck bis nahe an die Norm durch eine passend genähte Leibbinde zu erhöhen; nicht eine Gummibinde, da diese für die in Frage kommende Last der Eingeweide viel zu nachgiebig ist, sondern eine gut angepasste Binde aus derber fester Leinwand, zum Schnüren oder Binden eingerichtet. Bauchbinden passe ich stets selber an und zeichne die einzunähenden Falten überall selber vor, nur dann ist man sicher, dass der eben gewünschte Zweck in jedem Falle auch wirklich erreicht wird. Die Binde soll von unten nach oben drängen, somit unten enger sein: den etwa noch nöthig werdenden Gegendruck und Halt in der Oberbauchgegend kann dann ein Yos e angelegtes Corset mit der üblichen Bauchschneppe gewähren. Im Bund sind die Kleider nur ganz lose zu tragen, daher am. besten an Schulterträgern.

Kehren wir nach dieser kurzen durch Einschaltung der nöthigen Therapie bedingten Abschweifung zur Begutachtung der ätiologischen Momente zurück, so muss zunächst hervorgehoben werden, dass dem von Me issner betonten ,Mesohepar“ als primärer Bildungsanomalie nach der ganzen oben gegebenen Darstellung keine derartige und zumal keine ausschliessliche ätiologische Bedeutung beigemessen werden kann, ja dass dieses Motiv für den Causalnexus sowohl, als auch für die Entwickelung der secundären Erscheinungen nur von untergeordneter Bedeutung ist. Ist kein Hängebauch da, so bleibt die Leber am normalen Orte liegen, gleichviel wie lang die Ligamente sind. Ist aber erst Hängebauch vorhanden, dann gebrauchen die Ligamente allerdings nicht erst um so viel gedehnt werden, als sie überhaupt länger sind, als normal. Dies ist der einzige Effect eines präsumptiven „Mesohepar", dessen Annahme übrigens aus dem ganzen Sachverhalte durchaus nicht als ein nothwendiges Postulat herrorgeht.

Cantani, wenn er auch dem Causalnexus auf einer falschen Fährte nachgeht, ist dennoch mehr im Rechte, da er die Wan- 
derleber von vorausgegangenen Schwangerschaften abhängig macht. Nur sind seine Gründe nicht stichhaltig: 1) Der: Uterus habe während der Gravidität die Leber nach hinten gedrängt, 2) deren Ligamente gedehnt.

Uterus und Leber kommen selbst in den. letzten vier Wochen der Schwangerschaft nur selten in Contact: gewöhnlich besteht zwischen Leber und Uterus noch ein mehr oder minder breiter Streifen tympanitischen Schalles, und selbst wo dies nicht Statt hat, ist von einem Drucke keine Rede, sondern es ist nur höchstens ein einfacher leiser Contact vorhanden. Dass man aber nicht im Stande wäre, durch Percussion mit Sicherheit den unteren Leberrand zu bestimmen, ist ein nicht häufiger Fall.

Die Därme lagern hinter und oberhalb des Uterus. Der vom Uterus übrig gelassene Raum der Bauchhöhle ist etwa einem Kugelabschnitte zu vergleichen, dessen Basis die relativ feste, hintere Uteruswand ist, während die Oberfläche der Kugel durch das Zwerchfell repräsentirt wird. Denken wir uns einen analogen Fall als Experiment: ein hohler Kugelabschnitt mit fester Basis und schlaffem, nachgiebigem Mantel soll durch Einpumpen einer Flüssigkeit prall gefüllt werden. Wo wird sich die Spitze der Kugelwölbung bilden? Natürlich in der Verlängerung des auf dem Centrum der Basis stehenden Perpendikels. Wendet man dies auf das Zwerchfell an, so muss der Gipfel der Zwerchfellkuppel nach hinten verschoben werden. Der vordere Theil der Zwechfellkuppel wird sehr gedrückt, flach, der Gipfel selbst aber mag höher hinaufgedrückt werden, als er normaliter zu stehen pflegt. Der nächste Effect dieser Zwerchfellwölbung zeigt sich in der Verlagerung des Herzens. Das Herz, früher auf der Zwerchfellkuppel, somit auf einer nahezu horizontalen Ebene gelagert, gleitet jetzt bei der Lage auf einer steil abfallenden Ebene nach dem Fallgesetz nach vorn, und liegt in weiterem Umfange. der Brustwand an als früher. Daher findet man eine Verbreiterung des ganz matten Herzschalles ganz gewöhnlich bei vorgeschrittener Schwangerschaft als Symptom der veränderten Lage des Herzens, nicht aber ohne Weiteres als Zeichen einer Hypertrophie. Bezüglich der Leberdislocation kann ich keinen zwingenden Grund finden, der uns veranlassen sollte, daran zu zweifeln, dass die Leber durch den intraabdominellen Druck auch während der Gravidität eben so sicher in ihrer Lage fixirt werde, als sonst.

Für die Richtigkeit dieser Deduction, dass bei Schwangerschaft die abdominelle Druckrichtung nach oben und etwas hinten 
wirkt, spricht auch die Virchow'sche Erfahrung, dass bei Lebern mit Schnüratrophie der vordere, abgeschnürte Lappen mitunter nach oben umgeklappt wird, wobei leicht durch Knickung der Gallenblase hochgradige Gallenstauung zu Stande kommt.

Es ist bekannt, dass die Thoraxbasis bei Hochschwangeren breiter, aber weniger tief, in toto jedoch umfangreicher wird: unter solchen Umständen müsste die Kuppel des Diaphragma, falls letzteres ganz unverändert bliebe, tiefer herabgezerrt und flacher werden. Nun ist aber a priori undenkbar, dass am Zwerchfell während der Schwangerschaft nicht ebenso wie an Bauchdecken eine mehr oder weniger bedeutende Flächenvergrösserung zu Stande kommen sollte, so dass der Gipfel des Zwerchfells trotzdem in normalem, ja sogar über normalem Niveau stehend gedacht werden darf.

Sehen wir uns nach percutatorischen Belegen für dies Räsonnement um, - von anatomischen, die ganz fehlen, muss einfach abgesehen werden, - so finden wir einiges Material nur in einem Programm von Gerhardt: „De situ et magnitudine cordis gravidarum. Jena 18ă2. S. 19 finden wir 42 Bestimmungen der unteren Lungengrenze in der Lin. papill. bei Schwangeren. Gerhardt nennt dies "Stand des Diaphragma“. Diese Grenze war im oberen Rande der 6. Rippe $2 \mathrm{Mal}$ in 6. Rippe $25, \quad 36 \mathrm{Mal}$. im unteren Rande der 6. Rippe 9 ,

7. Rippe 5 ,

5. Rippe 1 ,

Somit 36 Mal unter 42 Fällen normal.

Diese Grenze, durch oberflächliche Percussion ermittelt, bezieht sich natürlich nur auf die vorderen Partien des Diaphragma; der Gipfel der Kuppel kann nur durch tiefen Anschlag bestimmt werden, was Frerichs z. B. in seinen Leberkrankheiten die sogenannte, ,wahre Lebergrenze" bestimmen heisst.

In meinen leider nur 10 Fällen ist die Lungengrenze etwa am gleichen Orte wie bei Gerhardt:
4. Spat. . . $1 \mathrm{Mal}$,
5. Cost. . . 1 ,
5. Spat. . . 3,
6. Cost. . . 3 , ,
7. Cost. . $\frac{2}{10}$

somit 6 Mal normal. 
Wahre Lebergrenze (Gipfel der Zwerchfellkuppe) in Lin. mam.:

$$
\begin{aligned}
& \text { 3. Spat. . . = } 1 \mathrm{Mal} \text {, } \\
& \text { 4. Cost. . . } 2 \text {," } \\
& \text { 4. Spat. - } 4 \text {, } \\
& \text { 5. Cost. . . } 1 \text { ", } \\
& \text { 6. Cost. . } \frac{=2}{10},
\end{aligned}
$$

somit 7 Mal übernormal.

Wenn auch die vorderen Partien des Zerchfelles normales Niveau beibehalten, so steigt also der Gipfel dennoch über die Norm hinauf. Dieser Gipfel, der für gewöhnlich vorn lagert, wo jetzt normales Niveau herrscht, muss somit mehr nach hinten verschoben und die Wölbung des Zwerchfelles somit in der oben theoretisch entwickelten Weise verändert sein. Denn es ist unmöglich, falls nicht Adhäsionen oder dergleichen bestehen, dass bei normal bleibender Lungengrenze die Kuppel des Zwerchfelles aufstiege, ohne ihren Ort zu wechseln, und zwar mehr nach hinten zu gehen. Demnach kann auch angenommen werden, dass das Zwerchfell eine nicht unbedeutende Dehnung i. e. Flächenvermehrung erlitten haben muss. Um alle diese Schlussfolgerungen sicher zu stellen, will ich beiläufig bezüglich der Sicherheit, mit welcher die ,wahre Lebergrenze" durch tiefe Percussion bestimmt werden kann, erwähnen, dass ich früher versuchsweise an Cadavern in diesen Grenzen tiefe Nadeln einstach, die dann gewöhnlich dass Zwerchfell durchbohrend, nachdem sie vielleicht noch ein Wenig die Leberoberfläche geritzt, gefunden wurden.

Die wahre Lebergrenze traf bei jenen Hochschwangeren in Lin. parast.

in Lin. axill.
die 3. Cost. 5 Mal,
"3. Spat. 1 ,
"4. Cost. 4 ,
i. e. 6 Mal übernormal.
7. Cost. . $1 \mathrm{Mal}$,
7. Spat. . . 5 ,
8. Cost. . . 1 ,
8. Spat. . 2 ,
9. Cost. . . 1 ,

somit stand auch in der Lin. parastern. der Gipfel des Zwerchfelles ziemlich hoch. War die Wölbung in oben erwähnter Weise verändert, demnach der hintere Rand der Leber gewissermaassen erhoben, so war zu erwarten, dass sich auch eine Zunahme der ganzen Leberhöhe in deren Projection auf die vordere Bauchwand markiren musste, was in der That der Fall war: 
Ganze Leberhöhe.
L. parast.
L. mam.
L. $a \times$ ill.

normal $(9-11 \mathrm{Cm}$.) $7 \mathrm{Mal}, \quad$ norm. $(11-13 \mathrm{Cm}$.) $3 \mathrm{Mal}, \quad$ normal $(12 \mathrm{Cm}) .2 \mathrm{Mal}$, darüber . . 3 Mal. darüber . . 7 Mal. über . . . 4 Mal, unter . . . 4 Mal:

Sollte eine Dislocation der Leber nach vorn angenommen werden dürfen, dann müsste eine Zunahme in der Höhe des ganz matten Schalles erkennbar sein. Das ist aber nicht der Fall:

\section{Ganz leerer Schall.}

Lin. parast. Lin. mam. Lin. axill. normal $(4-6 \mathrm{Cm}$.) $8 \mathrm{Mal}, \quad$ normal $(7-10 \mathrm{Cm}$.) $6 \mathrm{Mal}, \quad$ norm. $(7-10 \mathrm{Cm}$.) $0 \mathrm{Mal}$, über . . . . 2 Mal. unter . . . 4 Mal. unter . . . 10Mal.

Diese Zahlen ergeben sicher, dass wenigstens keine Dislocation nach vorn stattgefunden hatte, eher könnte man annehmen, dass die Leber ein wenig nach hinten in die Kuppel hineingeglitten wäre. Der Grund dazu wäre aber nicht mit Cantan i in dem directen Drucke des Uterus zu suchen, sondern vielmehr in der allgemeinen Druckrichtung der durch den schwangeren Uterus in ihrer Gestalt und Grösse wesentlich veränderten Bauchhöhle.

Immerhin gewinnt man keine ausgiebigen Anhaltspunkte für die Annahme, dass zwischen Gravidität als solcher und Dislocationen der Leber ein directer Causalnexus bestände.

Ich kann, wenn ich die ganze Summe von Thatsachen zusammenfasse, nur den schlaffen Hängebauch als directe Ursache der Wanderleber beschuldigen; und dieser Hängebauch giebt erst die Brücke ab, über welche Gravidität, weil sie fast die einzige, gewiss aber die allerhäufigste Ursache von Hängebauch ist, zur Wanderleber führt.

Der Causalnexus lautet, in seine einzelnen Etappen aufgelöst:

1) Gravidität und rechtzeitige Geburt.

2) Schlaffer Hängebauch.

3) Leberdescensus und Hepar pendens.

4) Demnach Dehnung der Bänder und Leberglitt nach vorn.

5) Endlich Zerrung und Torsion der in den Hilus hepatis eingehenden Stränge.

Zum Schlusse sei noch aufmerksam gemacht auf die in meinem Falle stets gleiche Gelegenheitsursache für den Eintritt der erwähnten Anfälle: es handelte sich dabei um Heben schwerer 
156 Winkler, Zur Casuistik und Aetiologie der Wanderleber.

Lasten in gebückter Körperhaltung. Dabei bildet die Wirbelsäule nicht nur nach hinten eine absolut feste Wand, sondern wirft einem Hohlspiegel gleich alle Kraftstrahlen direct nach vorn auf die schlaffen Bauchdecken. Das Diaphragma ist hierbei gleichfalls festgestellt in tiefer Inspirationsstellung mit Glottisverschluss, von unten, d. i. vom Becken her ist ebenfalls ziemlich fester Widerstand; somit wirkt der gesammte intraabdominelle. Druck auf die an und für sich schon schlaffen Bauchdecken, und weil gewöhnlich beim Ansetzen zum Heben diese ganze, eben auseinandergesetzte Situation mit einem Ruck zu Stande kommt, so ist einleuchtend, dass auch die bereits wie an schlaffen Bändern hängende Leber plötzlich nach vorn geschleudert werden muss. 